\title{
Pazopanib for Non-small Cell Lung Cancer: The First Case Report in Korea
}

\section{Jaemin Jo, MD ${ }^{1}$ \\ Jung Ho Kim, MD² \\ Ji Young Kim, $\mathrm{MD}^{1}$ \\ Changlim Hyun, $\mathrm{MD}^{3}$ \\ Jiyoung Rhee, $\mathrm{MD}^{1}$ \\ Jungmi Kwon, MD ${ }^{1}$ \\ Sanghoon Han, MD ${ }^{1}$ \\ Wookun Kim, MD ${ }^{1}$}

\section{${ }^{1}$ Department of Internal Medicine, Jeju National University Hospital, Jeju National University School of Medicine, Jeju, ${ }^{2}$ Department of Internal Medicine, Gachon University Gil Medical Center, Incheon, ${ }^{3}$ Department of Pathology, Jeju National University Hospital, Jeju National University School of Medicine, Jeju, Korea}

Correspondence: Sanghoon Han, MD Department of Internal Medicine, Jeju National University Hospital, Jeju National University School of Medicine, 15 Aran 13-gil, Jeju 63241, Korea

Tel: 82-64-754-8121

Fax: 82-64-717-1131

E-mail: btfulo@gmail.com

Received August 3, 2014

Accepted October 18, 2014

Published Online February 17, 2015

*Jaemin Jo and Jung Ho Kim contributed equally to this work.
Pazopanib is a potent multitargeted tyrosine kinase inhibitor that has been shown to have good efficacy in patients with renal cell carcinoma. A previous phase II trial demonstrated that short-term pazopanib administration was generally well tolerated and showed antitumor activity in patients with early-stage non-small cell lung cancer. Herein, we report on the case of a 66-year-old man with simultaneous metastatic squamous cell carcinoma of the lung and renal cell carcinoma who was treated with pazopanib. The patient showed an unexpected partial response and experienced a 10-month progression-free survival without significant toxicity. To the best of the authors' knowledge, this is the first report of pazopanib treatment in a non-small cell lung cancer patient in Korea. The results in this patient suggest that pazopanib may be a valid treatment option for advanced non-small cell lung cancer.

\section{Introduction}

Lung cancer is one of the most common cancers worldwide, and roughly $75 \%$ of patients have metastatic disease at the time of diagnosis [1]. However, aggressive cytotoxic treatment is rendered difficult because of poor performance status, multiple comorbidities, and advanced age. Therefore, there is significant interest in finding potent new chemother-

\author{
Key words \\ Lung neoplasms, Drug therapy, Tyrosine kinase inhibitor, \\ Pazopanib
}



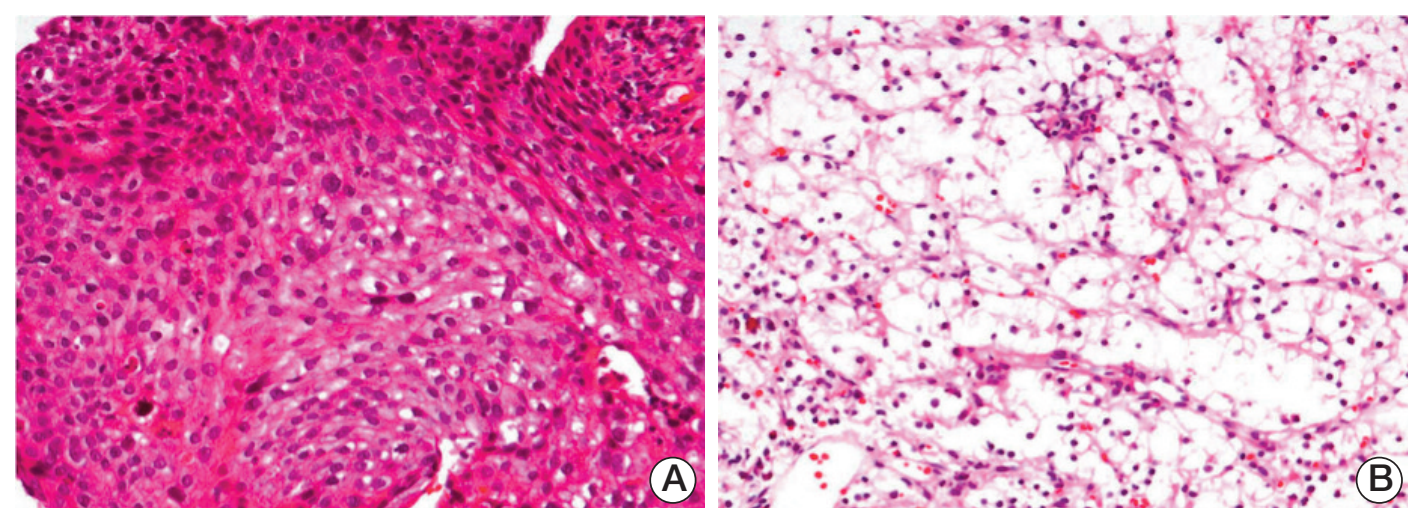

Fig. 1. Histological findings of the non-small cell lung cancer and renal cell carcinoma. (A) Lung: sheets or islands of large polygonal malignant cells with pink cytoplasm and distinct cell borders consistent with squamous cell carcinoma are observed (H\&E staining, $\times 200$ ). (B) Kidney: biopsy specimen of the renal mass showed alveolar growth of large polygonal cells with clear cytoplasm, uniform round nuclei, and inconspicuous nucleoli (H\&E staining, $\times 200)$. Neoplastic cells have clear cytoplasm and are arranged in nests with intervening blood vessels, consistent with clear cell carcinoma.
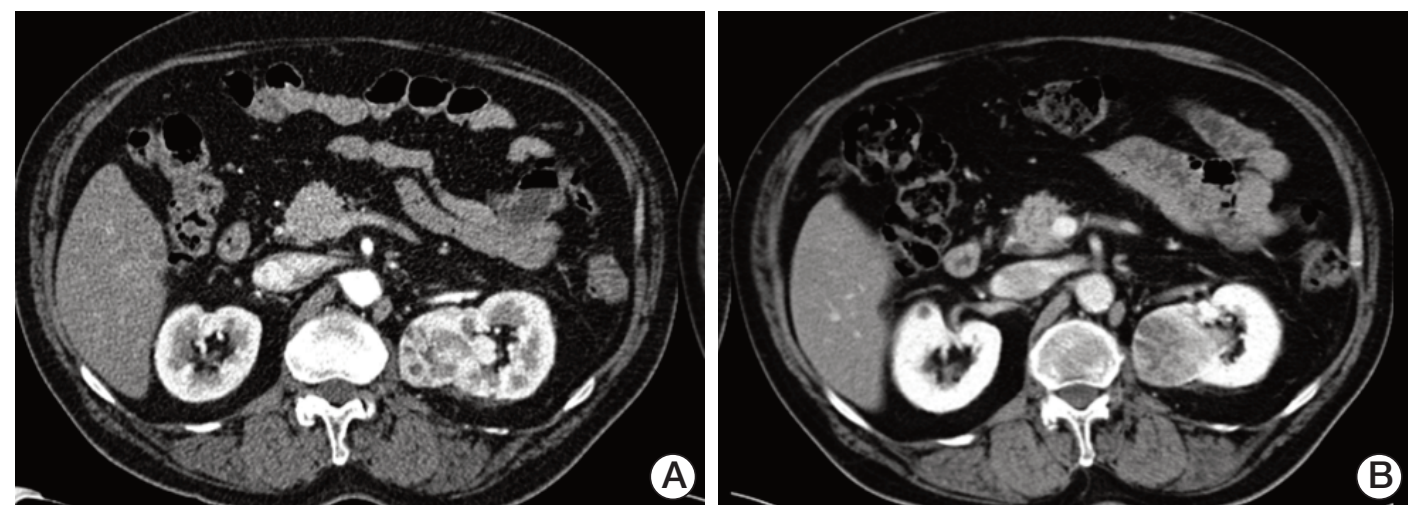

Fig. 2. Computed tomography findings of the renal cell carcinoma (RCC). (A) A lobulating contoured RCC mass measuring $5 \times 4 \mathrm{~cm}$ located in the left kidney is observed at the time of pazopanib initiation. (B) After 8 months of pazopanib treatment, the response of the RCC is considered to be stable disease $(5.5 \times 4.8 \mathrm{~cm})$.

Korea has not been reported. Herein, we report on the case of a NSCLC patient who achieved a partial response and 10-month progression-free survival (PFS) following pazopanib treatment.

\section{Case Report}

A 65-year-old man was admitted to the hospital with community-acquired pneumonia. Imaging studies, including computed tomography (CT), detected a mass in the left lower lobe of the lung. Further investigation revealed a mass measuring $5 \times 4 \mathrm{~cm}$ in the left kidney. Double primary cancers were suspected. Histological examination of a left bronchial biopsy specimen obtained by bronchoscopy resulted in a diagnosis of squamous cell carcinoma (Fig. 1). A needle biopsy was obtained from the mass in the left kidney, which enabled diagnosis of clear cell RCC (T1bN0M0) (Fig. 1). On chest $\mathrm{CT}$, multiple lung nodules indicative of metastatic NSCLC were observed in both sides of the lung. The patient declined surgical intervention for the RCC because of the presence of stage IV NSCLC. Thus, palliative chemotherapy for NSCLC was initiated. After one year of chemotherapy treatment consisting of four cycles of first-line gemcitabine- 

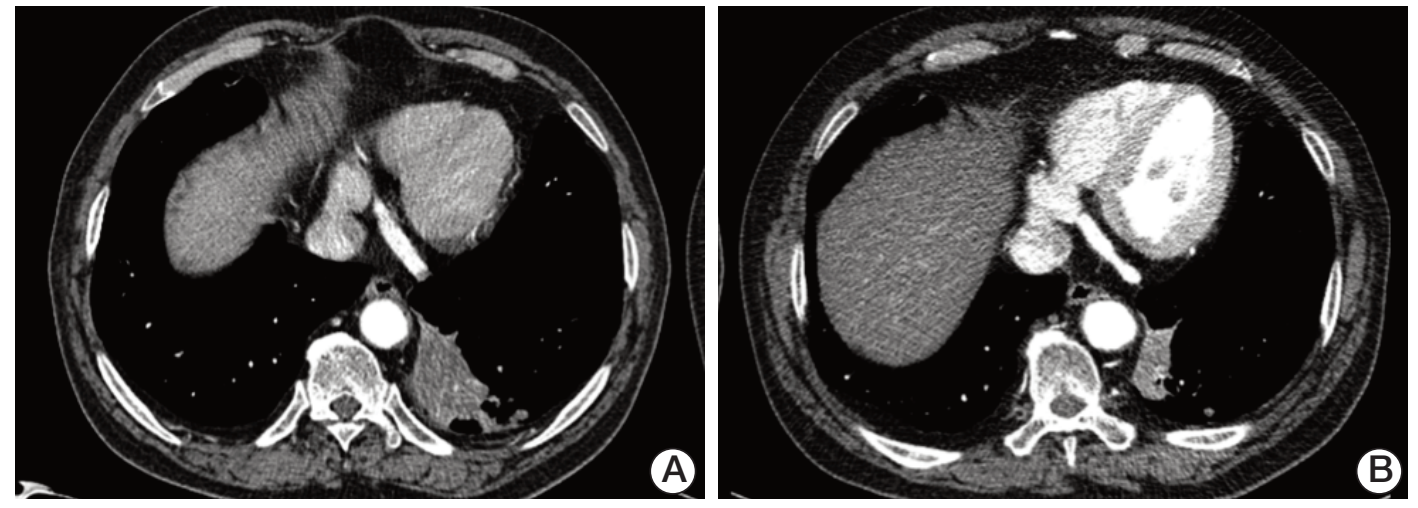

Fig. 3. Computed tomography findings of the squamous cell lung cancer. (A) A mass measuring $7 \times 3 \mathrm{~cm}$ is observed in the left lower lobe of the lung at the time of pazopanib initiation. (B) The mass decreases in size to approximately $3.5 \times 1.5 \mathrm{~cm}$ after 8 months of pazopanib treatment. The squamous cell cancer shows an unexpected partial response to pazopanib.

carboplatin and six cycles of second-line docetaxel, the NSCLC showed a partial response. However, the RCC showed progression of disease. Considering this, chemotherapy for the was suspended, and pazopanib ( $800 \mathrm{mg}$ per day) was initiated for the RCC.

At the time of pazopanib initiation, the patient, who was a former 100 pack-year smoker, was receiving an oral antihyperglycemic agent for type 2 diabetes. His blood pressure, pulse rate, and body temperature were all normal. He had good performance status without limitations of ordinary physical activity. Physical examination revealed no abnormal findings. Results of the laboratory tests were as follows: white cell count, 5,600/ $\mathrm{mm}^{3}$; hemoglobin, $13.0 \mathrm{~g} / \mathrm{dL}$; platelet count, 270,000/ $\mathrm{mm}^{3}$; albumin, $4.1 \mathrm{~g} / \mathrm{dL}$; aspartate aminotransferase (AST), $50 \mathrm{U} / \mathrm{L}$; alanine aminotransferase (ALT), $87 \mathrm{U} / \mathrm{L}$; alkaline phosphatase, $291 \mathrm{U} / \mathrm{L}$; blood urea nitrogen, $16.7 \mathrm{mg} / \mathrm{dL}$; serum creatinine, $1.0 \mathrm{mg} / \mathrm{dL}$; and urinalysis, normal.

At 8 months after initiation of pazopanib, the RCC had progressed slightly but was within the range of stable disease according to Response Evaluation Criteria in Solid Tumor ver. 1.1 (Fig. 2). However, the NSCLC showed an unexpected partial response (Fig. 3). During administration of pazopan$\mathrm{ib}$, the patient frequently experienced grade 2 elevations in AST and ALT, which improved within 1-2 weeks following therapy cessation. Despite this, pazopanib was well tolerated during the treatment period. At 10 months following initiation of pazopanib, both the NSCLC and RCC showed progression. Pazopanib was interrupted, and the patient received palliative chemotherapy with gefitinib for the NSCLC.

\section{Discussion}

Lung cancer is a leading cause of cancer death worldwide, and, despite the advent of several novel therapeutic approaches, only minimal improvements in survival have been achieved. Indeed, the 5-year survival rate is only approximately $15 \%$ in patients with lung cancer, and late diagnosis is considered to be a major contributor to poor prognosis [6]. As a result, most lung cancer patients are not candidates for surgery, and, thus, palliative chemotherapy is an important and widely adopted treatment option.

During the past decade, the development of novel agents, particularly molecular targeted agents and agents targeting pathways related to cancer pathophysiology, has led to innovated cancer therapy. In this regard, efforts to offer individualized therapeutic strategies based on the molecular characteristics of the tumor and patient are continuing. In advanced NSCLC, EGFR-targeting agents such as gefitinib and erlotinib have been shown to be beneficial in terms of overall survival and symptom control. Gefitinib and erlotinib are approved as first-line therapy in NSCLC patients with EGFR mutations [7,8]. However, development of resistance to these agents is a major clinical problem. The development of second- and third-generation EGFR tyrosine kinase inhibitors has been unsuccessful in overcoming resistance. In addition, the clinical applicability of these agents is limited by their lower efficacy compared with first-line agents. ErbB family members such as vascular endothelial growth factor receptor (VEGFR), platelet-derived growth factor receptor (PDGFR), fibroblast growth factor receptor (FGFR), and anaplastic lymphoma kinase are associated with the various stages of NSCLC pathogenesis, and agents targeting this 
family have shown promising results. Tumor angiogenesis plays a critical role in neoplastic growth and metastasis. Several VEGFR-targeting agents have been developed for inhibition of tumor angiogenesis and have demonstrated efficacy in several tumor types $[3,9]$. VEGFR is overexpressed in a large proportion of NSCLCs, and VEGFR and PDGFR levels have been shown to be correlated with lymph node metastasis and poor prognosis [10]. Thus, the antiangiogenesis agent bevacizumab, which targets VEGFR, has been used in combination with conventional chemotherapy for metastatic non-squamous cell lung cancer [11]. However, most patients will eventually experience disease progression due to the development of resistance.

Following the approval of bevacizumab, multitargeted antiangiogenesis agents were developed in an effort to overcome resistance to anti-vascular endothelial growth factor therapy due to compensatory upregulation of other angiogenic pathways, including those mediated by PDFGR and FGFR, and several trials using these agents for NSCLC have been initiated [10]. Pazopanib is a new oral antiangiogenesis agent that targets VEGFR, PDGFR, FGFR, and c-Kit through competitive inhibition of ATP. Pazopanib is mainly metabolized by CYP3A4 and eliminated slowly in the feces. It has been shown to have both therapeutic benefits and a favorable tolerability profile in patients with metastatic RCC, sarcoma, and other tumor types $[2,3,5,12,13]$. In an initial phase I clinical trial of pazopanib in advanced cancer patients, the most common adverse events were hypertension, diarrhea, hair depigmentation, and nausea. Abnormal AST, ALT, and bilirubin were reported in $38 \%, 24 \%$, and $13 \%$ of patients, respectively [14]. Altorki et al. [4], who conducted a phase II trial to investigate preoperative pazopanib monotherapy in early-stage NSCLC, found that $30(86 \%)$ and three $(8.6 \%)$ patients achieved preoperative volume reduction and partial response with short-term preoperative pazopanib treatment, respectively. Easily manageable grade 1-2 hypertension, diarrhea, fatigue, and elevated transaminase level were common adverse events.

In a phase $\mathrm{Ib}$ trial of concurrent pazopanib (400-800 mg once daily) and erlotinib (100-150 mg once daily) administration in 26 patients with various types of cancer, three $(12 \%$; all NSCLC) and 10 patients (38\%) had partial response and stable disease with a manageable toxicity profile, respectively [15]. To date, there is no solid evidence regarding the efficacy of new antiangiogenesis inhibitors for NSCLC, and it is possible that a yet undiscovered biomarker might help to improve treatment response in select patients. In the current case, pazopanib was not administered for treatment of lung cancer but rather for palliative treatment of RCC. Unexpectedly, the NSCLC showed a partial response and the patient achieved a 10-month PFS of the squamous cell carcinoma following pazopanib treatment. Pazopanib was well tolerated throughout the treatment period.

Patient outcome in our report is consistent with the outcomes reported in the phase II trial by Altorki et al. [4]. To the best of our knowledge, this is the first report on the use of pazopanib for treatment of lung cancer in Korea. Considering our findings, further study of pazopanib as a potential therapeutic agent for NSCLC is warranted.

\section{Conflicts of Interest}

Conflict of interest relevant to this article was not reported.

\section{References}

1. Mulshine JL, Sullivan DC. Clinical practice. Lung cancer screening. N Engl J Med. 2005;352:2714-20.

2. Sternberg CN, Davis ID, Mardiak J, Szczylik C, Lee E, Wagstaff J, et al. Pazopanib in locally advanced or metastatic renal cell carcinoma: results of a randomized phase III trial. J Clin Oncol. 2010;28:1061-8.

3. van der Graaf WT, Blay JY, Chawla SP, Kim DW, Bui-Nguyen B, Casali PG, et al. Pazopanib for metastatic soft-tissue sarcoma (PALETTE): a randomised, double-blind, placebocontrolled phase 3 trial. Lancet. 2012;379:1879-86.

4. Altorki N, Lane ME, Bauer T, Lee PC, Guarino MJ, Pass H, et al. Phase II proof-of-concept study of pazopanib monotherapy in treatment-naive patients with stage I/II resectable non-small-cell lung cancer. J Clin Oncol. 2010;28:3131-7.
5. Bible KC, Suman VJ, Molina JR, Smallridge RC, Maples WJ, Menefee ME, et al. A multicenter phase 2 trial of pazopanib in metastatic and progressive medullary thyroid carcinoma: MC057H. J Clin Endocrinol Metab. 2014;99:1687-93.

6. Ettinger DS, Akerley W, Bepler G, Blum MG, Chang A, Cheney RT, et al. Non-small cell lung cancer. J Natl Compr Canc Netw. 2010;8:740-801.

7. Fukuoka M, Wu YL, Thongprasert S, Sunpaweravong P, Leong SS, Sriuranpong V, et al. Biomarker analyses and final overall survival results from a phase III, randomized, openlabel, first-line study of gefitinib versus carboplatin/ paclitaxel in clinically selected patients with advanced non-small-cell lung cancer in Asia (IPASS). J Clin Oncol. 2011;29:2866-74.

8. Cappuzzo F, Ciuleanu T, Stelmakh L, Cicenas S, Szczesna A, 
Juhasz E, et al. Erlotinib as maintenance treatment in advanced non-small-cell lung cancer: a multicentre, randomised, placebo-controlled phase 3 study. Lancet Oncol. 2010;11:521-9.

9. Santoni M, Conti A, De Giorgi U, Iacovelli R, Pantano F, Burattini L, et al. Risk of gastrointestinal events with sorafenib, sunitinib and pazopanib in patients with solid tumors: a systematic review and meta-analysis of clinical trials. Int J Cancer. 2014;135:763-73.

10. Majem M, Pallares C. An update on molecularly targeted therapies in second- and third-line treatment in non-small cell lung cancer: focus on EGFR inhibitors and anti-angiogenic agents. Clin Transl Oncol. 2013;15:343-57.

11. Barlesi F, Scherpereel A, Rittmeyer A, Pazzola A, Ferrer Tur $\mathrm{N}$, Kim JH, et al. Randomized phase III trial of maintenance bevacizumab with or without pemetrexed after first-line induction with bevacizumab, cisplatin, and pemetrexed in advanced nonsquamous non-small-cell lung cancer: AVAPERL
(MO22089). J Clin Oncol. 2013;31:3004-11.

12. Ganjoo KN, Villalobos VM, Kamaya A, Fisher GA, Butrynski JE, Morgan JA, et al. A multicenter phase II study of pazopanib in patients with advanced gastrointestinal stromal tumors (GIST) following failure of at least imatinib and sunitinib. Ann Oncol. 2014;25:236-40.

13. Ahn HK, Choi JY, Kim KM, Kim H, Choi SH, Park SH, et al. Phase II study of pazopanib monotherapy in metastatic gastroenteropancreatic neuroendocrine tumours. Br J Cancer. 2013;109:1414-9.

14. Hurwitz HI, Dowlati A, Saini S, Savage S, Suttle AB, Gibson $\mathrm{DM}$, et al. Phase I trial of pazopanib in patients with advanced cancer. Clin Cancer Res. 2009;15:4220-7.

15. Dy GK, Infante JR, Eckhardt SG, Novello S, Ma WW, Jones SF, et al. Phase $\mathrm{Ib}$ trial of the oral angiogenesis inhibitor pazopanib administered concurrently with erlotinib. Invest New Drugs. 2013;31:891-9. 